\title{
Findings from landscape analysis in Cross River on pre- eclampsia/eclampsia
}

Population Council

Follow this and additional works at: https://knowledgecommons.popcouncil.org/departments_sbsr-rh

Part of the Demography, Population, and Ecology Commons, Family, Life Course, and Society Commons, International Public Health Commons, and the Maternal and Child Health Commons How does access to this work benefit you? Let us know!

\section{Recommended Citation}

"Findings from landscape analysis in Cross River on pre-eclampsia/eclampsia," Ending Eclampsia Research Brief. Washington, DC: Population Council, 2016. 


\section{ENDING \\ Eclampsia}

Despite global efforts to reduce preventable maternal and neonatal mortality, Nigeria's maternal mortality ratio is estimated at $\mathbf{5 7 6}$ deaths per 100,000 live births and neonatal death is estimated at 37 per 1,000 live births ${ }^{1}$.

Maternal and newborn deaths due to pre-eclampsia and eclampsia (PE/E) are preventable, yet in Nigeria this is the most significant direct cause of maternal deaths.

To appreciate the enormity of this problem at country and state levels, a landscape analysis was conducted by the Population Council in 2015 on PE/E in seven states in Nigeria. The main objectives of the landscape analysis were:

- To understand the level of programmatic and policy support for PE/E prevention and treatment;

- To analyze the gaps in providers' knowledge and competence in preventing, detecting, and managing PE/E;

- To determine primary health care (PHC) facilities' capacities to manage $\mathrm{PE} / \mathrm{E}$;

- To assess community awareness, beliefs, and experiences around $\mathrm{PE} / \mathrm{E}$;

- To understand the volume of research on PE/E in the last 15 years; and

- To determine priority areas for research and programmatic interventions around PE/E.

The Ending Eclampsia project seeks to expand access to proven, underutilized interventions and commodities for the prevention, early detection, and treatment of preeclampsia and eclampsia and strengthen global partnerships.

\section{PE/E IN BRIEF}

- Pre-eclampsia is a condition in pregnant women marked by an increase in blood pressure and protein in urine after 20 weeks gestation.

- Providing high quality antenatal care improves the prevention and early detection of pre-eclampsia and can prevent its progression to eclampsia.

- Eclampsia is a life-threatening condition characterized by convulsions in women with PE.

- Women in developing countries are 300 times more likely to die from eclampsia than women in developed countries.

- Prescribing low-dose aspirin and calcium to at-risk women can prevent pre-eclampsia and eclampsia.

- Pre-eclampsia and eclampsia can be managed by administering antihypertensive drugs and magnesium sulphate $\left(\mathrm{MgSO}_{4}\right)$.

- $\mathrm{MgSO}_{4}$ is the safest and most effective treatment for severe PE/E, and is one of 13 UN Life-Saving Commodities for Women and Children.

- PE/E and other hypertensive disorders in pregnancy increase the risk of pre-term births, which can lead to low birth weight, anemia, and stunting.

- Improved prevention, increased detection, and effective treatment of $P E / E$ can prevent unnecessary maternal and newborn deaths.

\section{IIII)}

Made possible by the generous support of the American people through the United States Agency for International Development (USAID).
The Population Council conducts research and delivers solutions that improve lives around the world. Big ideas supported by evidence: It's our model for global change.popcouncil.org

(c) 2015 The Population Council, Inc.
POPULATION COUNCIL 


\section{FACILITY CAPACITY \& PREPAREDNESS}

To assess institutional preparedness, researchers visited 11 facilities in Cross River State and recorded that four (36\%) of the facilities had guidelines available for management of pre-eclampsia, two (18\%) had all ANC equipment for the detection of $\mathrm{PE} / \mathrm{E}$ and five (46\%) use $\mathrm{MgSO}_{4}$ for the treatment of eclampsia.

\section{"QUOTATION. QUOTATION. QUOTATION. QUOTATION. QUOTATION. QUOTATION. QUOTATION. QUOTATION. QUOTATION."}

-POLICYMAKER, CROSS RIVER

During these facility assessments, researchers determined whether the facilities had the key ANC equipment required to detect pre-eclampsia, manage severe pre-eclampsia and eclampsia, and monitor for $\mathrm{MgSO}_{4}$ toxicity (figure 1).

FIGURE 1 Proportion of facilities with key equipment to detect $\mathrm{PE}$ and manage severe $\mathrm{PE} / \mathrm{E}$

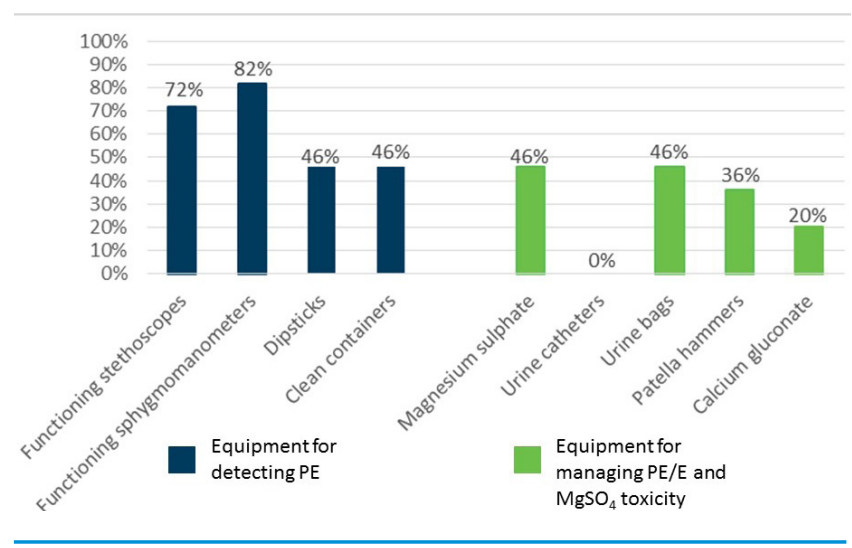

Four (36\%) facility managers reported always using $\mathrm{MgSO}_{4}$ to treat pre-eclampsia and eclampsia, one (9\%) said it is sometimes used, and six (55\%) reported that it is never used. When asked about how they obtain $\mathrm{MgSO}_{4}$ supplies at the facility, the Council found that $80 \%$ receive the drug as part of the regular central supply and $20 \%$ require their clients to purchase it from the market.

\section{PROVIDER KNOWLEDGE \& SKILLS}

Fifty-nine healthcare providers from Cross River participated in the study and answered questions about different aspects of PE/E prevention, detection, and treatment. Of the providers interviewed, $50.8 \%$ could correctly identify chronic hypertension in pregnancy, while $64 \%$ correctly identified signs and symptoms of pre-eclampsia, yet only $5 \%$ could identify severe preeclampsia, and $71 \%$ could correctly identify the signs and symptoms of eclampsia.

Researchers also assessed health care providers' knowledge of drugs used for preventing and managing $\mathrm{PE} / \mathrm{E}$ as well as calcium gluconate to treat $\mathrm{MgSO}_{4}$ toxicity (figure 2).

FIGURE 2 Provider knowledge of drugs for PE/E prevention and management $(n=59)$

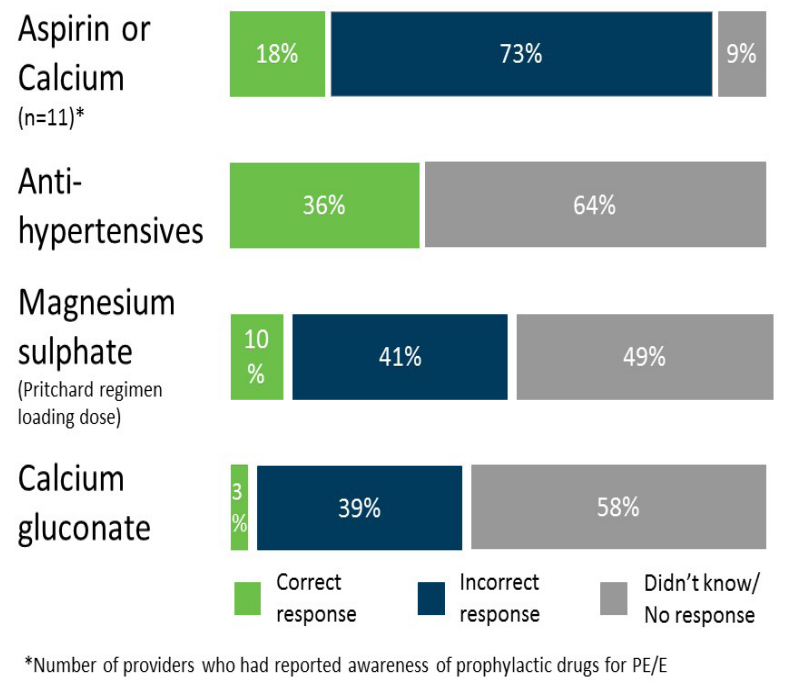

It is clear from the results, shown in figure 2, that providers are unaware of the prophylactic use of calcium and aspirin for women at risk of pre-eclampsia: only two (18\%) knew that anti-hypertensives (aldomet or nifepdipine) can be used to manage high blood pressure in pregnant women.

The Pritchard regimen for $\mathrm{MgSO}_{4}$ administration is considered the 'gold standard' for preventing and treating convulsions in severe $\mathrm{PE} / \mathrm{E}$, but only a small percentage of providers (10\%) could accurately describe the appropriate loading and maintenance doses of $\mathrm{MgSO}_{4}$.

Meanwhile, only three percent of providers could name the appropriate antidote for $\mathrm{MgSO}_{4}$ toxicity: calcium gluconate. 


\section{QUALITY OF CARE}

Quality of care was assessed through client provider observations along with client exit interviews. In Cross River, the Council observed 15 client-provider interactions during ANC visits and interviewed the same women after their consultations.

Clients should receive eight components of quality ANC care at each visit, and researchers asked the 15 clients whether their health provider completed each component during the consultation (figure 3 ).

FIGURE 3 Percent of clients who received all eight ANC components

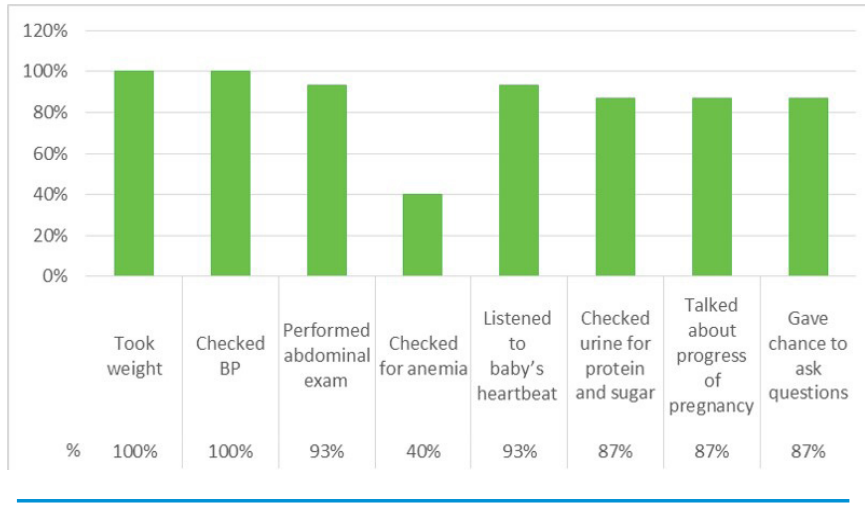

In addition to the eight essential components of ANC, questions and tests should be conducted to assess a woman's risk of developing $P E / E$, to detect $P E / E$, and inform clients of the signs of impending eclampsia.

During $75 \%$ of the observed ANC consultations, providers measured women's BP and checked urine for protein. None of the providers observed, however, performed the necessary checks to detect women at risk of developing PE. These included history of high blood pressure (BP) and diabetes, date of last delivery, client's parity, age and weight, edema of face, hands, legs, and ankles or advised the clients on the symptoms of impending eclampsia (severe headache, blurred vision, and pre-eclampsia with generalized body swelling).

\section{COMMUNITY KNOWLEDGE \& PERCEPTIONS}

The study also included in-depth interviews (IDIs) and focus group discussions (FGDs) for qualitative information from $P E / E$ survivors, community stakeholders, and families affected by $P E / E$. Misconceptions, myths, and mistrust between communities and health providers negatively influence care-seeking behaviors.
TEXT TEXT TEXT TEXT TEXT TEXT TEXT TEXT TEXT TEXT TEXT TEXT TEXT TEXT TEXT TEXT TEXT TEXT TEXT TEXT TEXT TEXT TEXT TEXT TEXT TEXT TEXT TEXT TEXT TEXT TEXT TEXT TEXT TEXT TEXT TEXT TEXT TEXT TEXT TEXT TEXT TEXT TEXT TEXT TEXT TEXT TEXT TEXT TEXT TEXT TEXT TEXT TEXT TEXT TEXT TEXT TEXT TEXT TEXT TEXT

\section{"QUOTATION QUOTATION QUOTATION QUOTATION QUOTATION QUOTATION QUOTATION QUOTATION"}

-FEMALE FGD PARTICIPANT, CROSS RIVER

\section{SURVIVORS' EXPERIENCES}

Interviews with survivors documented their careseeking pathways, including their PE/E experience, availability and accessibility of essential services and commodities, and the outcomes of the pregnancy for mother and child. Survivors' experiences provide insight informing strategies to work more closely with communities and health facilities improve access to, and use of, quality care.

The women interviewed were similar in age, ages at marriage and first pregnancy, and education.

TEXT TEXT

"QUOTATION."

-PE/E SURVIVOR, CROSS RIVER

\section{DISCUSSION}

This landscape analysis identified the gaps in facilities' and providers' capacities for preventing, detecting, and managing PE/E; assessing community awareness, beliefs, and experiences of PE/E; and it determined the gaps and priority areas for research and programs to improve access to prevention and treatment.

There are many areas of opportunity to prevent maternal death associated with $\mathrm{PE} / \mathrm{E}$ throughout pregnancy, and different approaches to make the most of these opportunities.

In Nigeria, all easily available anti-hypertensives for treatment of mild to moderate hypertension (aldomet and nifedipine) and severe hypertension (hydralazine) 
are cheap and obtainable, are often available in maternity emergency trays at most facilities and, if not, can be purchased externally. It is imperative that future interventions targeting providers, especially at primary and secondary facilities, include training on anti-hypertensives for pregnant women with PE/E.

For effective PE/E management, health facilities need to stock the necessary drugs and ANC equipment, and institutionalize guidelines for $\mathrm{PE} / \mathrm{E}$ treatment. Providers need training and re-training on detecting $P E / E$, and how and when to administer appropriate drugs (prophylactics, anti-hypertensives, $\mathrm{MgSO}_{4}$ ) to prevent and manage it. With few providers understanding these elements of $\mathrm{PE} / \mathrm{E}$ detection and treatment, it is not surprising these pregnancy complications account for more maternal deaths in Nigeria than any other direct cause, including postpartum hemorrhage ${ }^{2}$.

In addition to ensuring that health care providers are adequately trained to administer $\mathrm{MgSO}_{4}$ at the right time and with the proper doses, they also need to know the warning signs for $\mathrm{MgSO}_{4}$ toxicity and its antidote, calcium gluconate.

The final, essential component to reduce mortality from $\mathrm{PE} / \mathrm{E}$ is community awareness. Community members need to know the signs of $P E / E$ and understand the danger it poses for mothers and babies so they can seek medical care promptly.

\section{CONCLUSION}

Maternal and newborn deaths due to PE/E are preventable: by increasing community awareness of the condition, improving antenatal care quality, and scaling up proven best practices to prevent pre-eclampsia's escalation to severe pre-eclampsia and eclampsia. By detecting and managing pre-eclampsia, Ending Eclampsia can improve the survival rate of women and babies in Nigeria and other developing countries.

\section{RESOURCES}

1. National Population Commission, Federal Republic of Nigeria, and ICF International. (2014). Nigeria Demographic and Health Survey 2013.

2. Oladapo, O., Adetoro, O., et al. (2015). When getting there is not enough: a nationwide cross-sectional study of 998 maternal deaths and 1451 near-misses in public tertiary hospitals in a low-income country. BJOG: An International Journal of Obstetrics \& Gynaecology. 